\title{
Nearby star candidates in the Torino observatory parallax program
}

\author{
R. L. Smart ${ }^{1}$, M. G. Lattanzi ${ }^{1}$, H. Jahrei ${ }^{2}$, B. Bucciarelli ${ }^{1}$, and G. Massone ${ }^{1}$ \\ 1 Istituto Nazionale di Astrofisica (INAF), Osservatorio Astronomico di Torino, Strada Osservatorio 20, 10025 Pino Torinese, Italy \\ e-mail: [smart; lattanzi; bucc; massone]@oato.inaf.it \\ 2 Astronomisches Rechen-Institut am Zentrum für Astronomie der Universität Heidelberg, Mönchhofstr. 12-14, \\ 69120 Heidelberg, Germany \\ e-mail: hartmut@ari.uni-heidelberg.de
}

Received 16 September 2006 / Accepted 20 December 2006

\begin{abstract}
Aims. Candidates with suspect distances from the Catalog of Nearby Stars were included in the Torino Observatory Parallax Program with the goal to clarify their membership in that catalog.

Methods. Observations of the objects were made over the period 1996-2001 on the 1.05 m Torino telescope. The trigonometric parallaxes and proper motions were determined using standard techniques.

Results. Of the 22 objects examined, 11 are within the 25 pc horizon of the Catalog of Nearby Stars. The remaining objects are either misclassified, sub-dwarfs rather than main sequence dwarfs with consequently overestimated photometric distances, or objects with published trigonometric parallaxes that are incorrect.
\end{abstract}

Key words. astrometry - methods: data analysis - stars: distances - stars: fundamental parameters - subdwarfs stars: low-mass, brown dwarfs

\section{Introduction}

The Catalog of Nearby Stars (Gliese \& Jahreiß 1991, hereafter CNS) is an ongoing project to catalog all stars within 25 parsecs of the Sun. A comparison with Hipparcos results shows that it is complete to $M_{v}=9$ (Jahreiß \& Wielen 1997); however, in terms of distance, it was shown 12 years ago that the catalog was not complete to 8 pc (Henry 1994). Indeed it was a known shortfall that led the CNS group to work within 5 pc when, for example, constructing the luminosity function of nearby stars (Wielen et al. 1983). The original effort to build the CNS included all objects with either photometric or trigonometric parallax measurements greater than $0.0395^{\prime \prime}$ which, given the large errors on these estimates, leads to many stars being incorrectly included.

As of January 2006 the CNS had at least 206 refereed citations ranging from the study of white dwarfs to the structure of the Galaxy. The CNS is a unique tool in certain respects:

1) it provides a volume limited sample for galactic or statistic studies that is not dependent on magnitude or other parameter selection and their associated biases;

2) it allows a direct local determination of the luminosity and mass function for low mass stars;

3) since most objects have proper motions and distance estimates we can derive the local velocity dispersions;

4) it provides a target list for direct imaging of extrasolar planets and for the search for unseen companions such as $\mathrm{L}$ and T dwarfs;

5) it is also used extensively for studies of multiple star systems.

The strength of the CNS should be its reliability and completeness, therefore, efforts to improve those characteristics are very important. For these reasons in 1996 at the request of the CNS group 22 objects were added to the Torino Observatory Parallax Program (hereafter TOPP). The Torino program was in its 2 nd year with a target list of over 100 stars covering scientific interests of the local group: nearby red and white dwarfs, planetary nebulae, symbiotic stars, double stars; to these the requested objects were added and observed until 2001 when the CCD was changed. The 22 objects consisted of two samples: 1) possible erroneous objects, i.e. those CNS objects with the highest parallax errors and 2) faint LHS stars, i.e. objects with high proper motions but with no distance estimates. For many of this second sample the investigation was particularly interesting, because a combination of their photometric parallaxes and proper motions imply unrealistically large tangential velocities.

In this article we discuss the changes in observation and reduction procedures of the TOPP and our results for the two CNS samples.

\section{Observations and reduction procedures}

The observational and image reduction procedures are identical to those described in Smart et al. (1999) and the parallaxes were determined using the methods described in Smart et al. (2003, hereafter SMA03). In SMA03 the objects were of a color close to the field stars and we were able to ignore the Differential Color Refraction (DCR). In this work the targets are in general much redder than the field stars and the effect of DCR needs to be included.

To estimate the magnitude of the DCR we used the same procedure as used in Monet et al. (1992). To this end, we observed 9 fields on different nights for a range of hour angles and determined the correlation of the shift due to refraction with the color of the stars in the field. For the $I$ band in the $X$ coordinate (which is parallel to right ascension) we found the following relation:

$X_{\text {corrected }}=X_{\text {observed }}-8.1 *(V-I) * Z_{x}$ 
Table 1. Parallaxes and proper motions for the CNS candidates in the Torino Program. Columns are: target name, positions at J2000, number of reference stars, number of frames, proper motions, absolute parallax, correction applied to the relative parallax to obtain an absolute parallax, the interval of time for which we have observations, apparent magnitude in the $I$ band from in-house data unless discussed in Sect. 2, the CNS parallax along with the source.

\begin{tabular}{|c|c|c|c|c|c|c|c|c|c|c|}
\hline Name & $\begin{array}{c}\text { RA } \\
\text { h:m:s }\end{array}$ & $\begin{array}{c}\text { Dec } \\
\text { d:':" }\end{array}$ & $N_{*}, N_{\mathrm{f}}$ & $\begin{array}{r}\mu_{\alpha} \\
\text { mas/yr } \\
\end{array}$ & $\begin{array}{r}\mu_{\delta} \\
\text { mas/yr } \\
\end{array}$ & $\begin{array}{r}\pi \\
\text { mas } \\
\end{array}$ & $\begin{array}{r}\text { COR } \\
\text { mas } \\
\end{array}$ & $\begin{array}{c}\Delta T \\
\text { years } \\
\end{array}$ & $I$ & $\begin{array}{c}\pi_{\mathrm{CNS}}^{\text {source }} \\
\text { mas } \\
\end{array}$ \\
\hline LHS 1050 & $0: 15: 47$ & $+13: 33: 08$ & 8,81 & $606.3 \pm 1.5$ & $336.9 \pm 1.5$ & $89.0 \pm 3.5$ & 1.84 & 5.07 & 10.04 & $86.6 \pm 13.4^{Y}$ \\
\hline GD 806 & $1: 12: 28$ & $-01: 51: 19$ & 11,64 & $14.9 \pm 1.4$ & $-73.7 \pm 1.4$ & $8.4 \pm 3.3$ & 2.04 & 5.41 & 13.55 & $99.0 \pm 11.0^{w}$ \\
\hline GJ 3140 & $2: 10: 14$ & $+11: 32: 11$ & 8,170 & $-20.0 \pm 0.6$ & $3.6 \pm 0.8$ & $5.3 \pm 1.8$ & 2.56 & 5.41 & 9.85 & $90.0 \pm 30.0^{p}$ \\
\hline LP 475-70 & $4: 25: 53$ & $+12: 11: 47$ & 20,67 & $-102.1 \pm 1.1$ & $-232.8 \pm 1.1$ & $59.4 \pm 2.8$ & 1.35 & 5.31 & 14.77 & $81.0 \pm 11.0^{w}$ \\
\hline LHS 1675 & $4: 31: 07$ & $-05: 20: 26$ & 11,39 & $-175.0 \pm 2.1$ & $-507.8 \pm 2.7$ & $28.5 \pm 3.6$ & 1.83 & 5.32 & 11.00 & $87.0 \pm 10.2^{H}$ \\
\hline $\mathrm{Cl}^{*} \mathrm{Mel}^{a}$ & $4: 35: 00$ & $+17: 33: 40$ & 17,31 & $3.4 \pm 1.8$ & $-18.0 \pm 1.5$ & $2.7 \pm 3.8$ & 1.62 & 5.24 & 12.55 & $94.0 \pm 17.0^{p}$ \\
\hline LP 60-179 & 9:02:51 & $+68: 3: 18$ & 13,48 & $182.9 \pm 1.4$ & $345.3 \pm 2.0$ & $85.3 \pm 3.8$ & 2.47 & 5.25 & 9.75 & $98.0 \pm 10.6^{Y}$ \\
\hline LHS 295 & $10: 52: 04$ & $+13: 59: 51$ & 11,71 & $-1119.0 \pm 1.1$ & $206.8 \pm 1.6$ & $80.2 \pm 2.7$ & 1.90 & 5.37 & 9.95 & $83.9 \pm 25.7^{Y}$ \\
\hline GJ 3667 & 11:29:19 & $+18: 16: 35$ & 10,55 & $6.2 \pm 1.2$ & $6.5 \pm 1.1$ & $1.3 \pm 2.9$ & 2.21 & 6.09 & 13.23 & $114.0 \pm 13.0^{w}$ \\
\hline LHS 2472 & 11:54:08 & $+09: 48: 56$ & 5,38 & $89.6 \pm 2.0$ & $-796.7 \pm 1.9$ & $82.7 \pm 4.4$ & 2.06 & 5.13 & 9.92 & $96.1 \pm 19.7^{Y}$ \\
\hline GJ 1167a & 13:09:36 & $+28: 59: 10$ & 5,15 & $-337.5 \pm 2.4$ & $-203.1 \pm 2.3$ & $83.1 \pm 6.3$ & 2.04 & 4.22 & 11.14 & $86.0 \pm 12.5^{H}$ \\
\hline LHS 369 & $14: 20: 09$ & $-09: 36: 35$ & 8,32 & $-607.0 \pm 1.2$ & $-826.8 \pm 1.9$ & $68.1 \pm 3.8$ & 1.85 & 6.15 & 10.25 & $91.1 \pm 15.0^{Y}$ \\
\hline LHS 3872 & $22: 54: 45$ & $-05: 28: 37$ & 6,32 & $610.1 \pm 1.6$ & $354.4 \pm 2.1$ & $40.4 \pm 4.9$ & 2.18 & 5.09 & 11.21 & $94.0 \pm 23.0^{H}$ \\
\hline
\end{tabular}

Source: $Y=$ Yale Parallax catalog, $w=$ photometric parallax assuming white dwarf with the McCook \& Sion (1999) calibration, $p=$ photometric parallax assuming main sequence star, $H=$ Parallax from Heintz (1994), $a=\mathrm{Cl}^{*}$ Mel. is Cl* Melotte $25 \mathrm{LH} 27$.

where the positions are in mas, $V-I$ is the difference of the $V$ and $I$ magnitudes - e.g. the color of the object and $Z_{x}$ is the projected tangent of the zenith distance in the $X$ direction. In this article all UBVRI magnitudes are in the Johnson-Cousins system as defined in Bessel (1990b, Table 6). The constant 8.1 has an error of 3.6 and is a function of temperature and pressure, hence this is for the mean conditions (mean temperature $5^{\circ}$ Celsius, mean pressure $710 \mathrm{mmHg}$ ) at Torino.

As an independent check we considered the work of Stone (2002) where relations are given for combinations of $B, V, R$ and $I$ magnitudes. This used a very different calibration technique that compares the instantaneous stellar positions in wide and narrow band filters. It is assumed that the DCR in the narrow band filter is negligible as the sampling of the energy distribution is so small that spectral type variations will not induce significant atmospheric shifts. By comparison of the stellar positions the DCR can be evaluated, this procedure is more precise as the temporal atmospheric changes do not play a role. The relation for DCR to $V-I$ was not given but in a private communication Stone (2003) provided the following:

$X_{\text {corrected }}=X_{\text {observed }}+1.6-6.1 *(V-I) * Z_{x}$

for a temperature of $10.9^{\circ}$ Celsius and pressure of $581.8 \mathrm{mmHg}$. This relation, given the temperature and pressure differences between Flagstaff and Torino, is practically identical in the color and hour angle range in which the TOPP operates. Even though this effect is small and it makes no difference to the solution, as testing on a few fields shows, we include Eq. (1) as part of the pipeline to guard against possible systematic errors.

We have not made photometric observations for all fields: when we do not have in-house photometry we have taken the best $V$ and $I$ magnitudes from the literature and applied the DCR correction to just the target star. In SMA03 we used $I$ band photometry to calculate the correction from measured relative parallax to astrophysically useful absolute parallax using a model of the Galaxy. When we do not have any photometry available, we use any field stars from the literature with $I$ band magnitudes to calibrate the instrumental magnitudes of the first frame, assuming no color terms. The resulting corrections from relative to absolute parallax are listed in the respective tables. The objects with literature values are: LHS 1050, LHS 369 and GJ 1167a (Bessell 1990a), LHS 356 (Leggett 1992), LHS 370 (Dawson \& Forbes 1992), LHS 3872 (Weis 1996), LHS 1675 (Eggen 1987), and finally LHS 318 and LHS 326 were estimated by interpolating adjacent Torino fields during a good night. The DCR correction as defined by Eq. (1) provides a zero correction at $(V-I)=0$, since the mean $(V-I)$ is in general between .5 and 1.5 this means applying the correction only to the target stars leads to an "overcorrection". Since the objects with literature values are all redder that this mean value, e.g. with $(V-I)>2$, it can be seen from Eq. (1) that it still worthwhile applying this correction. This is obviously not an optimum situation and photometric observations are ongoing to cover all the sequences.

\section{Sample 1: possible erroneous entries}

In Table 1 we report the TOPP results for stars with poor parallaxes in the CNS. Note that as discussed in SMA03 the proper motions are essentially relative not absolute hence any use of these proper motions should be done with care.

Of the 13 targets in this sample, 8 have parallaxes larger than the CNS limit (39.5 mas) and 5 are significantly smaller. Since the CNS sources are quite varied, we now discuss objects individually.

\subsection{LHS 1050, LP 60-179, LHS 295, LHS 2472, and GJ $1167 a$}

These stars all have CNS parallaxes that agree with ours to within the error; we therefore only confirm their presence in the CNS and add our measurements to the literature values.

\section{2. $G D 806$}

The object entered into the CNS due to an original classification of DA wk white dwarf which would imply a distance of 10 pc (Bues 1984, private communication). Further work indicated that it was more likely to be a metal poor subdwarf with a companion (Bues \& Rupprecht 1985). The parallax determined in the TOPP indicates a distance of over $100 \mathrm{pc}$, supporting the 
new interpretation. The proper motions found using GSC2.3 material (McLean et al. 2000) are (10.5, -91.7) mas/yr, very similar to ours and indicative of an object at a large distance.

\subsection{GJ 3140}

The object entered into the CNS following the assignment of spectral type M4 by Robertson (1984, star 115) which would give a photometric distance of $11 \mathrm{pc}$. Subsequent spectral classification in the Palomar/MSU nearby-star spectroscopic survey (Hawley et al. 1996) found a spectral type of M4 III and a distance of $5 \mathrm{kpc}$. The parallax found here indicates a distance of $188 \mathrm{pc}$ and the proper motions are significantly non-zero. Both these results are inconsistent at the 3 sigma level with expectations for either a M4V or a M4III, this is an interesting object that deserves more attention.

\section{4. $L P 475-70$}

As discussed in SMA03, this object was listed in the CNS as a white dwarf with a photometric parallax of 81 mas, we find 59 mas still within the $25 \mathrm{pc}$ limit but further away. Probably the difference in distance is due to errors in the photometry and the use of a spectrophotometric calibration that appears to overestimate absolute magnitudes (SMA03) or it may be due to an undetected companion.

\subsection{LHS 1675}

In Heintz (1994) this object is attributed a parallax of $86 \pm$ 10 mas, much larger than our value of $28.5 \pm 3.6$ mas. However, Heintz added the remark: "is fainter than cataloged and now near the sky background", obviously to explain the large error. In the revised proper motion of Bakos et al. (2002) this object is attributed a proper motion of $(0.031,-0.629)$ which, even given the caveats previously mentioned on proper motions, is not reconcilable with our value $(-.175,-.507)$. Indeed, the original Luyten measurement $(-.188,-.486)$ and the measurement by Salim \& Gould (2003,) of $(-.163,-.505)$ are more consistent with ours. Since the selection of POSS plates by Bakos et al. in this case have an epoch difference of only 7 years, we assume that it is their value in error ${ }^{1}$.

\section{6. $\mathrm{Cl}^{\star}$ Melotte $25 \mathrm{LH} 27$}

Photographic infrared photometry indicated that this object was at a distance of $10 \mathrm{pc}$ (Leggett \& Hawkins 1988). Reid (1992) found a proper motion of $(0.016,-0.021)$ implying a larger distance. Combining apparent magnitudes from Reid with 2MASS magnitudes in the M-dwarf photometric distance determination of Henry et al. (2004, hereafter HEN04) we obtain a distance of $12 \mathrm{pc}$, incompatible with our parallax. We therefore conclude this is a background $\mathrm{K} / \mathrm{M}$ giant rather than an $\mathrm{M}$ dwarf which would be consistent with the proper motion and our parallax.

\subsection{GJ $3667=W D 1126+185$}

This object has already been discussed in SMA03. Putney (1997) classified this object as a $\mathrm{DC}+\mathrm{G} / \mathrm{K}$ binary however, in a recent article by Farihi et al. (2005) they conclude that this is probably a

\footnotetext{
1 See also the discussion in Hambly et al. (2004) regarding the reliability of the Bakos et al. catalog.
}

$\mathrm{sdB}+\mathrm{G} / \mathrm{K}$ binary. This interpretation was developed to make the spectra, color and apparent magnitude compatible, however, it is also consistent with the near zero parallax found in the TOPP.

\subsection{LHS 369}

In the Yale Catalog (van Altena et al. 1995) this object is reported as having a parallax of $91.1 \pm 15.0$ mas. In Jao et al. (2005) it was remeasured and a parallax of $71.52 \pm 1.38$ mas found, in agreement with our value.

\subsection{LHS 3872}

The CNS parallax for this object is $94.0 \pm 23.0$ mas from Heintz (1994) while the TOPP estimate, $40.6 \pm 4.9$ mas, is significantly different. Again, Heintz explained its large parallax error by the remark "is fainter than cataloged, and now at $\delta=-6^{\circ}$ almost below the brightened sky". The photometric parallax using the calibration of HEN04 with photometry from Weis (1996) and infrared photometry from the 2MASS catalog is 37.8 mas, in agreement with our value.

\section{Sample 2: faint LHS stars}

In Table 2 we report the TOPP results for faint LHS stars. In addition, we include the derived tangential velocities using TOPP parallaxes and proper motions.

To facilitate discussion we provide in Table 3 the BVRIJHK photometry and resulting photometric parallaxes from the HEN04 calibration. Objects that are of a thick disk or halo origin usually have tangential velocities larger than $200 \mathrm{~km} \mathrm{~s}^{-1}$ and photometric distances that overestimate the ones derived from trigonometric parallaxes. However, as recent work on the determination of Galaxy component (thin, thick or halo) membership has shown (Reid et al. 2001; Spagna et al. 2004), velocities and photometric parallaxes are not sufficient for this discrimination.

To help classify these objects we compared their properties to the sample of normal M-Dwarfs from HEN04 and the sub and extreme sub M dwarfs from Gizis (1997). To make this comparison we constructed reduced proper motion diagrams and color magnitude diagrams. We found that the combination of $V$ and $I$ gave the best separation of the three types and in Figs. 1 and 2 we plot the HEN04 and Gizis samples along with the TOPP results. Here we include the $V, I$ magnitude combination for illustrative purposes, however the other magnitude combinations were also examined in parallel.

\subsection{LHS 269, 306 and 370}

These objects all have parallaxes that put them within the $25 \mathrm{pc}$ boundary of the CNS. Their transverse velocities, while large, still allow a thin disk origin and hence a main sequence luminosity class. The photometric parallaxes obtained from the HEN04 calibration are compatible. Scholz et al. (2005) combining photometric parallaxes with proper motion for LHS 269 find a larger velocity and hence halo/thick disk origin. Based on the measured trigonometric parallax and the photometric distance consistency, and the location in both the HR and reduced proper motion diagrams with main sequence $\mathrm{M}$ dwarfs, it is probably more likely to be a disk object. LHS 269 lies slightly above the main sequence which may mean that it is a binary system, however the images and solution showed no evidence for a companion. 
Table 2. TOPP results for the faint LHS stars. Columns are target name, positions, number of reference stars, number of frames, proper motions, absolute parallax, correction applied to the relative parallax to obtain an absolute parallax, the interval of time for which we have observations, apparent magnitude in the $I$ band, tangential velocity based on TOPP results.

\begin{tabular}{|c|c|c|c|c|c|c|c|c|c|c|}
\hline $\begin{array}{l}\text { Name } \\
\text { LHS }\end{array}$ & $\begin{array}{c}\text { RA } \\
\mathrm{h}: \mathrm{m}: \mathrm{s}\end{array}$ & $\begin{array}{c}\text { Dec } \\
\mathrm{d}:^{\prime}:^{\prime \prime}\end{array}$ & $\overline{N_{*}, N_{\mathrm{f}}}$ & $\begin{array}{r}\mu_{\alpha} \\
\operatorname{mas} / \mathrm{yr}\end{array}$ & $\begin{array}{r}\mu_{\delta} \\
\mathrm{mas} / \mathrm{yr} \\
\end{array}$ & $\begin{array}{r}\pi \\
\text { mas } \\
\end{array}$ & $\begin{array}{r}\text { COR } \\
\text { mas } \\
\end{array}$ & $\begin{array}{r}\Delta T \\
\text { years }\end{array}$ & $I$ & $\begin{array}{r}V_{\mathrm{T}} \\
\mathrm{km} \mathrm{s}^{-1} \\
\end{array}$ \\
\hline 269 & $9: 29: 14$ & $+25: 58: 17$ & 16,23 & $-1046.4 \pm 2.1$ & $-267.5 \pm 2.3$ & $54.1 \pm 4.3$ & 2.13 & 3.93 & 12.65 & $104.5 \pm 8.2$ \\
\hline 279 & 10:09:17 & $+35: 14: 55$ & 10,58 & $-704.0 \pm 1.2$ & $-1118.0 \pm 1.0$ & $28.4 \pm 2.7$ & 2.04 & 4.29 & 12.76 & $235.4 \pm 22.2$ \\
\hline 306 & 11:31:08 & $-14: 57: 14$ & 8,11 & $416.7 \pm 3.2$ & $-1368.7 \pm 4.3$ & $83.4 \pm 8.5$ & 1.79 & 4.24 & 10.94 & $81.5 \pm 8.3$ \\
\hline 318 & 11:56:53 & $+26: 40: 55$ & 9,12 & $602.0 \pm 1.7$ & $-1217.7 \pm 2.5$ & $24.8 \pm 6.0$ & 2.40 & 4.95 & 13.00 & $266.0 \pm 64.9$ \\
\hline 326 & $12: 24: 26$ & $-04: 43: 35$ & 13,54 & $-1142.9 \pm 1.6$ & $-636.1 \pm 1.6$ & $11.7 \pm 4.3$ & 2.37 & 4.21 & 13.00 & $531.7 \pm 197.4$ \\
\hline 334 & $12: 34: 15$ & $+20: 37: 08$ & 8,34 & $332.5 \pm 1.3$ & $-1296.5 \pm 1.9$ & $22.4 \pm 4.1$ & 2.39 & 5.26 & 15.01 & $284.3 \pm 52.5$ \\
\hline 356 & :17 & $+35: 01: 02$ & 8,22 & $-1161.5 \pm 2.3$ & $-314.2 \pm 1.4$ & $11.1 \pm 5.8$ & 2.91 & 5.13 & 13.00 & $622.8 \pm 327.4$ \\
\hline 360 & & $+05: 43: 34$ & 11,32 & $-768.6 \pm 1.1$ & $-847.7 \pm 1.6$ & $17.5 \pm 3.4$ & 2.01 & 4.16 & 13.36 & $311.5 \pm 60.5$ \\
\hline 370 & $14: 20: 53$ & $+36: 57: 16$ & 8,39 & $-1338.5 \pm 1.8$ & $234.6 \pm 1.3$ & $50.3 \pm 3.0$ & 2.02 & 3.91 & 12.85 & $159.2 \pm 9.4$ \\
\hline
\end{tabular}

Table 3. Johnson-Cousins BVRI and 2MASS JHK photometry and derived photometric parallaxes based on HEN04. All " $r$ " band magnitudes are taken from the literature; objects labeled with an “*” have BVI photometry taken from the literature as described in Sect. 2; other BVI magnitudes are found from in-house photometry.

\begin{tabular}{lcccccccr}
\hline \hline LHS & $B$ & $V$ & $R$ & $I$ & $J$ & $H$ & $K$ & $\pi_{\mathrm{P}}$ \\
\hline & & & & & & & & \\
LHS 269 & 18.16 & 16.80 & 14.76 & 12.65 & 10.906 & 10.310 & 9.958 & $43.6 \pm 6.8$ \\
LHS 279 & 16.59 & 15.95 & 14.66 & 12.76 & 11.333 & 10.842 & 10.609 & $17.6 \pm 2.7$ \\
LHS 306 & 16.10 & 14.52 & 12.58 & 10.94 & 9.359 & 8.764 & 8.497 & $72.9 \pm 10$. \\
LHS 318* & 16.10 & 15.51 & - & 13.00 & 12.503 & 11.983 & 11.797 & $5.6 \pm 1.2$ \\
LHS 326* & 15.80 & 14.53 & - & 13.00 & 11.927 & 11.430 & 11.234 & $5.6 \pm 1.0$ \\
LHS 334 & 19.70 & 17.69 & - & 15.01 & 13.746 & 13.252 & 13.044 & $4.8 \pm 0.8$ \\
LHS 356* & 17.20 & 16.37 & - & 13.00 & 13.307 & 12.877 & 12.621 & $4.7 \pm 1.2$ \\
LHS 360 & 16.00 & 15.28 & 14.28 & 13.36 & 12.390 & 11.849 & 11.662 & $5.1 \pm 0.8$ \\
LHS 370 & 17.20 & 16.17 & 14.67 & 12.85 & 11.054 & 10.553 & 10.254 & $30.4 \pm 4.6$ \\
\hline
\end{tabular}

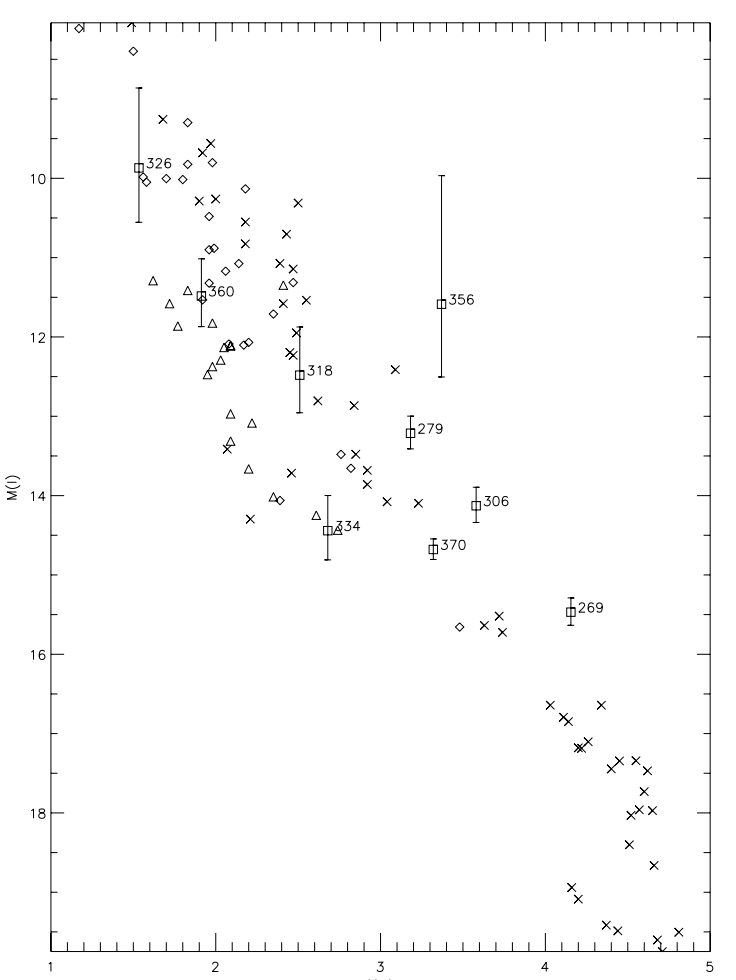

Fig. 1. The H-R diagram for $M$ dwarfs, squares with error bars and LHS numbers are TOPP results, crosses main sequence dwarfs from HEN04, diamonds sub-dwarfs and triangles extreme sub-dwarfs from Gizis (1997).

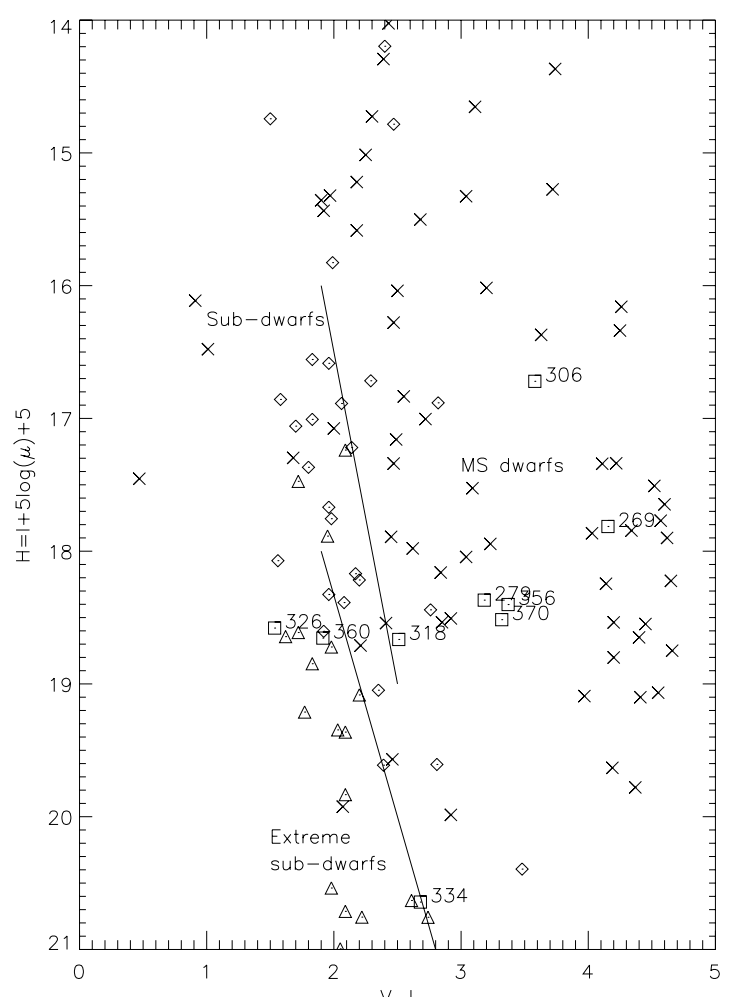

Fig. 2. The reduced proper motion diagram for $M$ dwarfs, symbols as in Fig. 1. Regions of M-dwarf sub-type (dwarf, sub-dwarf, extreme subdwarf) are indicated. 


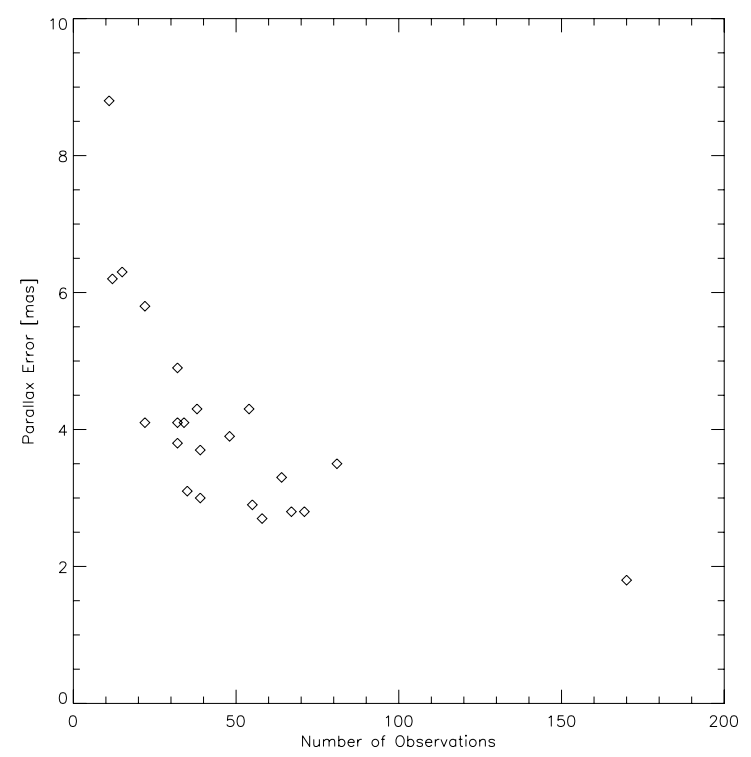

Fig. 3. Correlation between the number of observations and the resulting parallax error.

\subsection{LHS 279, 318, 326, 334, 356, and 360}

These objects have parallaxes that put them beyond the boundary of the CNS. Based on their location in the HR and reduced proper motion diagram, LHS 326, 334 and 360 are almost certainly sub or more likely extreme-sub M dwarfs, while LHS 279 and 356 are probably normal M dwarfs. LHS 318 is unclear but at most a sub-dwarf. The object LHS 360 is reported in Costa et al. (2006) with a parallax measurement of $10.33 \pm 2.85$ mas which is within 2 sigma of our value, they also conclude that this object is probably sub-dwarf in nature. Considering the tangential velocities the main discrepancy is with LHS 356; however, it also has the largest relative error of over $50 \%$. In addition, its position in the HR diagram indicates that the parallax is over estimated; a reduction of the parallax to move the object closer to the main sequence would result in a equal reduction in the velocity. These targets are choice candidates for spectroscopic follow-up to fully understand the intrinsic properties, manifested by the HR diagram position, and the kinematical properties, manifested in the reduced proper motion diagram position.

\section{Conclusions}

We have determined parallaxes for 22 CNS candidates, and we have confirmed 11 to be within the $25 \mathrm{pc}$ limit. No objects were resolved as binaries in the frames we have analyzed, however, faint companions could still be present.

The median error in the parallaxes presented here is 3.9 mas and the median error in proper motion $1.6 \mathrm{mas} / \mathrm{yr}$. This error is higher than that expected for the general TOPP program because these targets were given a low priority, hence the number of observations per target is in general low. Although the number of observations is not the only parameter that modulates the error in the final parallax determination, the number of reference stars, magnitude of the target star, and length of observational sequence, are also important. A plot of number of observations vs. parallax error (Fig. 3) shows a direct and expected correlation.
TOPP is an ongoing project. The program has recently upgraded the CCD and an automated control system has been introduced to improve the efficiency of the observational program. We have begun observing a new target list and expect to complete the reduction of the previous target list in the coming year.

Acknowledgements. We wish to thank the referee, Dr. Todd Henry, for numerous comments and suggestions that improved this article. This publication makes use of data products from the Two Micron All Sky Survey, which is a joint project of the University of Massachusetts and the Infrared Processing and Analysis Center/California Institute of Technology, funded by the National Aeronautics and Space Administration and the National Science Foundation.

This research has made use of the VizieR catalogue access tool, CDS, Strasbourg, France.

The Guide Star Catalogue II is a joint project of the Space Telescope Science Institute and the Osservatorio Astronomico di Torino. Space Telescope Science Institute is operated by the Association of Universities for Research in Astronomy, for the National Aeronautics and Space Administration under contract NAS5-26555. The participation of the Osservatorio Astronomico di Torino is supported by the Istituto Nazionale di Astrofisica.

\section{References}

Bakos, G. Á., Sahu, K. C., \& Németh, P. 2002, ApJS, 141, 187

Bessell, M. S. 1990a, A\&AS, 83, 357

Bessell, M. S. 1990b, PASP, 102, 1181

Bues, I., \& Rupprecht, G. 1985, Ap\&SS, 110, 163

Costa, E., Méndez, R. A., Jao, W.-C., et al. 2006, AJ, 132, 1234

Dawson, P. C., \& Forbes, D. 1992, AJ, 103, 2063

Eggen, O. J. 1987, AJ, 93, 379

Farihi, J., Becklin, E. E., \& Zuckerman, B. 2005, ApJS, 161, 394

Gizis, J. E. 1997, AJ, 113, 806

Gliese, W., \& Jahreiß, H. 1991, On: The Astronomical Data Center CD-ROM: Selected Astronomical Catalogs, Vol. I, ed. S. G. Gesser, \& L. E. Brotzmann (NASA/Astronomical Data Center, Goddard Space Flight Center, Greenbelt, MD)

Hambly, N. C., Henry, T. J., Subasavage, J. P., Brown, M. A., \& Jao, W.-C. 2004, AJ, 128, 437

Hawley, S. L., Gizis, J. E., \& Reid, I. N. 1996, AJ, 112, 2799

Heintz, W. D. 1994, AJ, 108, 2338

Henry, T. J. 1994, in Cool Stars, Stellar Systems, and the Sun, ASP Conf. Ser., 64,569

Henry, T. J., Subasavage, J. P., Brown, M. A., et al. 2004, AJ, 128, 2460

Jahreiß, H., \& Wielen, R. 1997, in Hipparcos - Venice '97, ESA SP-402, 675

Jao, W.-C., Henry, T. J., Subasavage, J. P., et al. 2005, AJ, 129, 1954

Leggett, S. K. 1992, ApJS, 82, 351

Leggett, S. K., \& Hawkins, M. R. S. 1988, MNRAS, 234, 1065

McCook, G. P., \& Sion, E. M. 1999, ApJS, 121, 1

McLean, B. J., Greene, G. R., Lattanzi, M. G., \& Pirenne, B. 2000, in Astronomical Data Analysis Software and Systems IX, ed. N. Manset, C. Veillet, \& D. Crabtree, ASP Conf. Ser., 216, 145

Monet, D. G., Dahn, C. C., Vrba, F. J., et al. 1992, AJ, 103, 638

Putney, A. 1997, ApJS, 112, 527

Reid, I. N., Sahu, K. C., \& Hawley, S. L. 2001, ApJ, 559, 942

Reid, N. 1992, MNRAS, 257, 257

Robertson, T. H. 1984, AJ, 89, 1229

Salim, S., \& Gould, A. 2003, ApJ, 582, 1011

Scholz, R.-D., Meusinger, H., \& Jahreiß, H. 2005, A\&A, 442, 211

Smart, R. L., Bucciarelli, B., Lattanzi, M. G., Massone, G., \& Chiumiento, G. 1999, A\&A, 348, 653

Smart, R. L., Lattanzi, M. G., Bucciarelli, B., et al. 2003, A\&A, 404, 317

Spagna, A., Carollo, D., Lattanzi, M. G., \& Bucciarelli, B. 2004, A\&A, 428, 451 Stone, R. C. 2002, PASP, 114, 1070

van Altena, W. F., Lee, J. T., \& Hoffleit, E. D. 1995, The General Catalogue of Trigonometric Stellar Parallaxes, Fourth edition (Yale University Observatory)

Weis, E. W. 1996, AJ, 112, 2300

Wielen, R., Jahreiß, H., \& Krüger, R. 1983, in Nearby Stars and the Stellar Luminosity Function, ed. A. G. D. Philip, \& A. R. Upgren, IAU Colloq., 76,163 